\title{
Soft projectile impact forces measurement using Hopkinson bars: application to ice, artificial bird and rubber
}

\author{
Ramón del Cuvillo, Jose Alfonso Artero-Guerrero, Jesús Pernas-Sánchez, Jorge López \\ Puente \\ Department of Continuum Mechanics and Structural Analysis, University Carlos III \\ Madrid \\ Avda. De la Universidad, 30, 28911 Leganés, Spain
}

\begin{abstract}
This work presents an experimental campaign of impacts of soft projectiles to measure the induced force during the impact. Three different materials acting as soft impactors that could strike against a aeronautical structural component: ice, artificial bird and rubber have been impacted at several velocities against an aluminium Hopkinson bar. This device has been instrumented with semiconductor strain gauges that allow to obtain the induced compression strain. Additionally, all the impacts were recorded using high-speed video cameras, allowing the kinematic analysis of the projectile during the impact. After the results study, it has been concluded that there is a linear dependency between the kinetic energy and the peak force for all three materials. Added to that, it has been proved that the higher peak force corresponds to ice, despite the kinetic energy, followed by rubber and finally the artificial bird. In addition, while ice and artificial bird projectiles get radially dispersed after the impact, rubber spheres rebound due to its different behaviour. The obtained data is of great interest to design structures which could be subjected to impacts of soft materials such as aeronautic structures
\end{abstract}

\section{Introduction}

Aeronautical transportation industries are continuously improving and optimizing their structures with the goal of obtaining more reliable and lightweight components. Among the known loads, impacts events could drive the design of some of the parts such as the wings leading edges. Several articles could be found about the effect of impacts on aeronautic structures[1], [2] for instance the authors of the current work have publish several works related to the low and high velocity impact of rigid projectiles on composite laminates [3], [4].

Impactors could be classified using different strategies, being one of them related to its strength (soft and hard bodies). Regarding the hard bodies, many efforts have been done in order to understand the influence of the different parameters such as mass or shape when impacting lightweight structures [2], [4]. Soft body impactors have received less attention even if are quite common in the aeronautic industry, and hence are worth to be studied. Its 
behaviour its more complex compared to hard bodies, because they deform considerably during the impact and increases the number of variables which has to be considered such as its resistance and how they fail. In almost all the works found in the literature the characteristic of the projectile have been analysed separately: velocity, mass, shape, etc. [5][9], but it was not possible to find any work in which different materials have been considered. The techniques used in these studies were based on impact momentum [8], load cells [9] and stress waves propagation using modified Hopkinson devices [6], [7].

\section{Experimental procedure}

In this section, the manufacturing process of the projectiles is presented; in addition the experimental setup and the methodology proposed to evaluate the force during the impact of soft bodies is explained.

\subsection{Projectiles preparation procedure}

The present study focusses on the comparison of three different materials considered as soft impactors projectiles. Ice, artificial bird and natural rubber are manufactured with different techniques, but with an identical shape to avoid geometrical effects. The selected one is a sphere of $40 \mathrm{~mm}$ of diameter.

- In order to obtain the ice spheres, the manufacturing process was similar to the one presented in detail in a previous work of the authors [9]. In this work, it is explained the necessity of a previous ice piece, frozen directionally to obtain an air bubble free block. Once this block is frozen the desired projectile shape was obtained using two pre-warmed moulds which melts and carves the block into the desired shape. With this process, the average mass of projectiles is $26.9 \pm 1.3 \mathrm{~g}$ with an average density of $800 \pm 40 \mathrm{~kg} / \mathrm{m}^{3}$.

- The projectiles of artificial bird were obtained from a more complex manufacturing process. As other authors have done previously to assure repeatability, projectiles made of gelatine are chosen [6], [10]. The recipe presented in the work of PernasSanchez et al. [6] has been used, where the detailed process is shown. The most important steps are the addition of gelatine powder to cold water in a $10 \%$ percentage, stirring to mix them, and then 10 minutes rest. The following step is to warming the mixture between $45^{\circ}$ and $55^{\circ}$ and finally cool it down to $40^{\circ}$ before the pouring. In contrast to ice sphere, where the final shape cannot be directly obtained, gelatine mixture can be poured in a 3D printed mould with the desired shape. The final average mass is $33.8 \pm 1.2 \mathrm{~g}$ and an average density of $1010 \pm 36 \mathrm{~kg} / \mathrm{m} 3$.

- Natural rubber spheres are manufactured by a company "Cauchos Payvi", with the specific dimensions and the highest density and lowest deviation: $1220 \pm 10 \mathrm{~kg} / \mathrm{m}^{3}$. The average mass is $40.9 \pm 0.33 \mathrm{~g}$.

\subsection{Impact test}

The final objective of the study is to evaluate the impact force and behaviour of the abovementioned materials in a wide range of impact velocity. All three materials are evaluated with the same methodology explained below. 
In order to accelerate the projectiles, a gas gun was used. The spheres were guided inside an $18 \mathrm{~m}$ long tube with $60 \mathrm{~mm}$ of inner diameter. To save the distance between the sphere and the tube, a 3D printed sabot was used, which has been specially designed to get separated from the impactor sphere after the barrel mouth. At the end of the barrel, a laser sensor was placed to measure the velocity of the projectile. The velocity is proportional to the air pressure inside a vessel placed behind the tube, that can be regulated in order to obtain similar kinetic energies. For ice, velocities in range between $127 \mathrm{~m} / \mathrm{s}$ and $183 \mathrm{~m} / \mathrm{s}$ were measured. For artificial bird they were between $117 \mathrm{~m} / \mathrm{s}$ and $175 \mathrm{~m} / \mathrm{s}$ and for rubber $81 \mathrm{~m} / \mathrm{s}$ and $154 \mathrm{~m} / \mathrm{s}$.

In order to measure the impact force, the projectile impacts against a 3 meter solid aluminium bar with higher diameter $(50 \mathrm{~mm})$, where the compression wave induced by the impact will travel and get measured using strain gauges. In the present study, four strain gauges were placed at $300 \mathrm{~mm}$ from the impact face, forming a Wheatstone bridge. It has to be noted that the strain gauges employed are manufactured with semiconductor materials, so they allow to increase the system sensitivity. The strain gauges were connected to a highspeed data acquisition system (Dewetron DEWE-800) with a sampling frequency of $1 \mathrm{MHz}$.

Additionally, impacts were recorded with a high-speed camera placed perpendicular to the bar, in order to visualize and analyse the impact behaviour. All the tests were recorded with a 1024x1024 pixel resolution, and at 20000 frames per second. Combining the obtained images with a tracking software, it is possible to obtain the spread of the projectiles over the impact area.

\section{Experimental results}

In order to evaluate ice, artificial bird and rubber behaviour at high velocities impact, both compression strain waves and recorded videos have been used. Figure 1 shows an example for each material impacting against the Hopkinson solid bar. To compare impacts with similar kinetic energies, chosen velocities were $126 \mathrm{~m} / \mathrm{s}$ for the gelatine projectile $(269 \mathrm{~J}), 136 \mathrm{~m} / \mathrm{s}$ for ice $(249 \mathrm{~J})$ and $113 \mathrm{~m} / \mathrm{s}$ for rubber $(261 \mathrm{~J})$. No matter the material, it can be observed a sudden quasi-linear increase when the compression wave reaches the strain gauges, followed by a reduction of the slope just before reaching the peak strain. Afterwards, the strain begins to decrease softly until reaching the 0 value.

During compression, some differences between the materials are appreciated. On one side, the maximum strain reached for the gelatine impact is $\sim 135 \mu \varepsilon$, whereas, for ice and rubber this value is approximately $175 \mu \varepsilon$, despite the higher kinetic energy of the gelatine impact. On the other side, the period of the compression waves is almost equal for gelatine and ice (near $0.4 \mathrm{~ms}$ ), whereas the wave promoted by the rubber needs more time to get back to the unloaded state $(0.6 \mathrm{~ms})$. These observations can be extrapolated to the rest of the tests performed.

Assuming elastic behaviour in the bar, the stress and force can be calculated knowing elastic modulus and cross area (74858 $\mathrm{MPa}$ and $1963.5 \mathrm{~mm}^{2}$ respectively), since the stress induced by the impacts is much lower than the yield stress. Comparing the peak force of the different projectiles at different impact energies, some conclusions can be extracted:

- Ice impacts result on a higher peak force than rubber or gelatine in the selected range at constant kinetic energy. Lowest peak forces are found for gelatine impacts. 
- As an indicator of the repeatability, the mean square error $\mathrm{R}^{2}$ is also calculated for each material. All three are below 10\% dispersion, but rubber spheres are the most reproducible $\left(\mathrm{R}^{2}=0.99\right)$ followed by gelatine $\left(\mathrm{R}^{2}=0.96\right)$ and ice $\left(\mathrm{R}^{2}=0.93\right)$. This conclusion is associated with the manufacturing process.

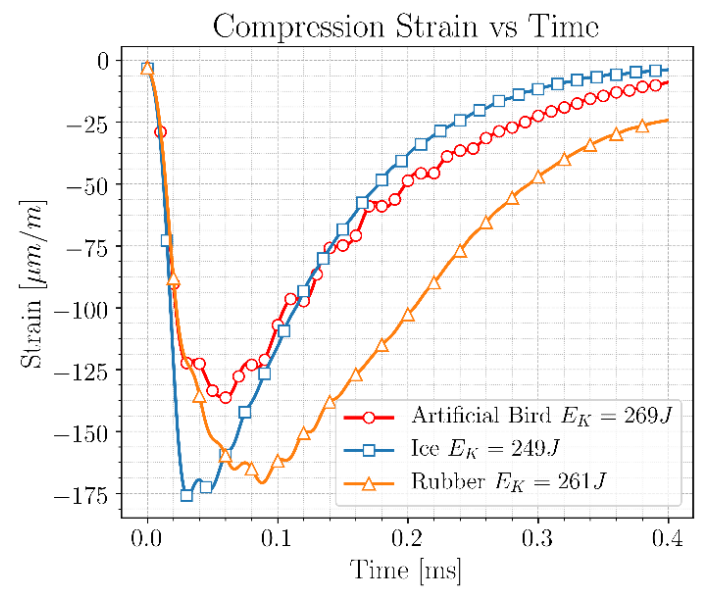

Figure 1: Ice, Artificial Bird and Natural Rubber spheres impact at similar kinetic energies.

Figure 2 shows a sequence obtained from the high-speed video camera for an ice impact at $136 \mathrm{~m} / \mathrm{s}$. In these images, as the same authors have observed before [9], the ice sphere loses its transparency due to internal cracks and fragmentation. Correlating the images and the curve obtained from the strain gauges, it can be observed that the peak force occurs just between the two first pictures, when the sphere is fragmented and internally cracked.

Even if the same observation can be extrapolated for all the ice impacts, it is not the same for the other materials. While ice is internally cracked and fragments are radially dispersed, gelatine spheres present no internal damages during the impact. Their behaviour is similar to a viscous fluid flowing as a water when it jets against a rigid wall. This behaviour is in accordance with other authors [6], [10]. Finally, rubber spheres behaviour is completely different. Their high hyperelastic behaviour makes the projectiles rebound, and allows the investigators to recover it with almost the original shape.

Therefore, it has been analysed only the radial spreading for ice and gelatine impactors. By means of a tracking software, the diameter of the spread can be measured following the front of the dispersed material. The results of this measurement for an ice impact at $136 \mathrm{~m} / \mathrm{s}$ and an artificial bird impact at $135 \mathrm{~m} / \mathrm{s}$ are collected in Table 1. From these results, it can be observed that for both materials, the radial dispersion increases with time. On the one hand, ice spread reaches almost 6 times the initial diameter of the sphere during the first $0.2 \mathrm{~ms}$, whereas gelatine spread area is much lower, with half spread diameter at the same time. In any case, when the spread area overpasses the recorded area it is no possible to measure it.

The previous observations and conclusions can be extrapolated to the other impacts. Where higher the velocity, higher normalized diameter will be measured for the same time. 


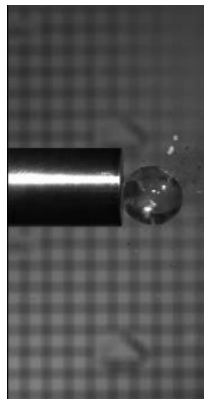

$\mathrm{t}=0 \mathrm{~ms}$

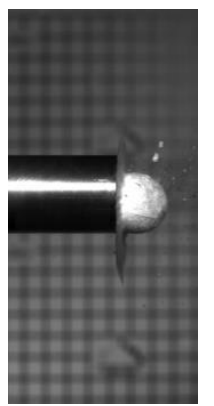

$\mathrm{t}=0,05 \mathrm{~ms}$

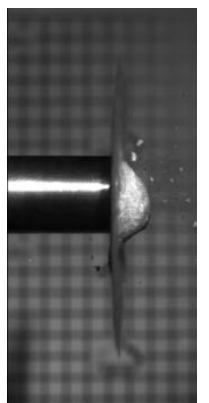

$\mathrm{t}=0,2 \mathrm{~ms}$

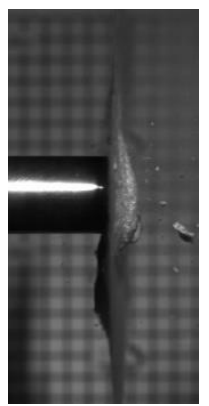

$\mathrm{t}=0,4 \mathrm{~ms}$

Figure 2: Ice impact at $v=136 \mathrm{~m} / \mathrm{s}(20000 \mathrm{fps})$

Table 1. Spread area measured with a tracking software for an ice impact at $136 \mathrm{~m} / \mathrm{s}$ and an artificial bird impact at $135 \mathrm{~m} / \mathrm{s}$.

\begin{tabular}{|c|c|c|}
\hline Time (ms) & $\begin{array}{c}\text { Ice Normalized } \\
\text { Diameter }\end{array}$ & $\begin{array}{c}\text { Artificial Bird } \\
\text { Normalized Diameter }\end{array}$ \\
\hline 0 & 1 & 1 \\
\hline 0,05 & 2,445 & 1,078 \\
\hline 0,1 & 3,645 & 2,041 \\
\hline 0,15 & 4,883 & 2,270 \\
\hline 0,2 & 5,905 & 2,830 \\
\hline 0,25 & 6,908 & 3,637 \\
\hline
\end{tabular}

\section{Conclusions}

In this work an analysis of the impact of three different materials considered as soft impactors at high velocities is performed. Ice, gelatine and rubber spheres have been impacted against an aluminium Hopkinson bar at similar kinetic energies between $200 \mathrm{~J}$ and $500 \mathrm{~J}$. The induced compression wave has been measured with semiconductor strain gauges, that allow a high sensitivity. Knowing the properties and dimensions of the Hopkinson bar, stress and forces can be easily calculated. Also a high-speed camera has been employed in order to record the impact and allow a subsequent tracker analysis.

From the compression waves measured, it can be concluded that ice and gelatine have similar impact time, whereas ice has always a higher peak force. On the other side, rubber presents higher impact time, but similar peak forces to ice. High speed videos recorded can be correlated with the compression waves measured in terms of impact time. An ice impact will take $0.4 \mathrm{~ms}$ to impact completely according to the video and the compression wave. Thus, the internal cracks will cover the whole sphere before $0.05 \mathrm{~ms}$ and the peak force is found before this time.

Finally, while rubber spheres have a hyperplastic behaviour, and rebound during the impact, ice and gelatine projectiles are completely dispersed. The spread area of the impacts has been measured thanks to a tracking software. From these measures it can be concluded that the covered area by ice is much higher than for gelatine, being more than double the diameter for the same time. Whereas, ice spread is made of small fragments and gelatine 
spread is a continuous flow due to the viscous fluid behaviour. This difference should be taken into account for secondary impacts.

\section{References}

[1] D. Fernández-Fdz, J. López-Puente, and R. Zaera, 'Prediction of the behaviour of CFRPs against high-velocity impact of solids employing an artificial neural network methodology', Composites Part A: Applied Science and Manufacturing, vol. 39, no. 6, pp. 989-996, Jun. 2008, doi: 10.1016/j.compositesa.2008.03.002.

[2] T. Mitrevski, I. H. Marshall, R. Thomson, R. Jones, and B. Whittingham, 'The effect of impactor shape on the impact response of composite laminates', Composite Structures, vol. 67, no. 2, pp. 139-148, Feb. 2005, doi: 10.1016/j.compstruct.2004.09.007.

[3] J. A. Artero-Guerrero, J. Pernas-Sánchez, J. Martín-Montal, D. Varas, and J. LópezPuente, 'The influence of laminate stacking sequence on ballistic limit using a combined Experimental/FEM/Artificial Neural Networks (ANN) methodology', Composite Structures, vol. 183, no. 1, 2017, doi: 10.1016/j.compstruct.2017.03.068.

[4] J. A. Artero-Guerrero, J. Pernas-Sánchez, J. López-Puente, and D. Varas, 'Experimental study of the impactor mass effect on the low velocity impact of carbon/epoxy woven laminates', Composite Structures, vol. 133, pp. 774-781, 2015, doi: 10.1016/j.compstruct.2015.08.027.

[5] R. Hedayati and S. Ziaei-Rad, 'A new bird model and the effect of bird geometry in impacts from various orientations', Aerospace Science and Technology, vol. 28, no. 1, pp. 9-20, Jul. 2013, doi: 10.1016/j.ast.2012.09.002.

[6] J. Pernas-Sánchez, J. Artero-Guerrero, D. Varas, and J. López-Puente, 'Artificial bird strike on Hopkinson tube device: Experimental and numerical analysis', International Journal of Impact Engineering, vol. 138, p. 103477, Apr. 2020, doi: 10.1016/J.IJIMPENG.2019.103477.

[7] J. D. Tippmann, H. Kim, and J. D. Rhymer, 'Experimentally validated strain rate dependent material model for spherical ice impact simulation', International Journal of Impact Engineering, vol. 57, pp. 43-54, Jul. 2013, doi:

10.1016/j.ijimpeng.2013.01.013.

[8] F. Allaeys, G. Luyckx, W. Van Paepegem, and J. Degrieck, 'Characterization of real and substitute birds through experimental and numerical analysis of momentum, average impact force and residual energy in bird strike on three rigid targets: A flat plate, a wedge and a splitter', International Journal of Impact Engineering, vol. 99, pp. 1-13, Jan. 2017, doi: 10.1016/j.ijimpeng.2016.08.009.

[9] J. Pernas-Sánchez, J. A. Artero-Guerrero, D. Varas, and J. López-Puente, 'Analysis of Ice Impact Process at High Velocity', Exp Mech, vol. 55, no. 9, pp. 1669-1679, Nov. 2015, doi: 10.1007/s11340-015-0067-4.

[10] M. A. Lavoie, A. Gakwaya, M. N. Ensan, D. G. Zimcik, and D. Nandlall, 'Bird's substitute tests results and evaluation of available numerical methods', International Journal of Impact Engineering, vol. 36, no. 10, pp. 1276-1287, Oct. 2009, doi: 10.1016/j.ijimpeng.2009.03.009. 